\title{
A Molecular Dynamics Simulation of the Tensile Behavior of Y-Branched-CNT/SiC Nanocomposite
}

\author{
Guobin Zhenga*, Hideaki Sano $^{\mathrm{b}}$, Osamu Nakagoe ${ }^{\mathrm{c}}$, Shuji Tanabe ${ }^{\mathrm{d}}$ \\ ${ }^{1}$ Division of Chemistry and Materials Science, Graduate School of Engineering, Nagasaki \\ University, Nagasaki, 852-8521, Japan \\ agbzheng@nagasaki-u.ac.jp, bsano@nagasaki-u.ac.jp, cn-osamu@nagasaki-u.ac.jp, \\ ds-tanabe@nagasaki-u.ac.jp
}

Keywords: Molecular dynamics; carbon nanotube; SiC; nanocomposite.

\begin{abstract}
The purpose of incorporating CNTs into ceramic materials is to enhance the toughness of ceramic materials, in which the interface plays a key role. Due to the nanoscale of nanocomposites, however, it is not easy to acquire a direct knowledge of the interface behavior. In this research, we simulated the dynamics of $\mathrm{CNT} / \mathrm{SiC}$ and branched $\mathrm{CNT} / \mathrm{SiC}$ under uniaxial tensile stress using molecular dynamics method (LAMMPS). The simulation method using molecular dynamics provide an insight into designing an effectively toughened ceramic nanocomposite materials.
\end{abstract}

\section{Introduction}

Carbon nanotube and graphenes have attracted broad attention as reinforcement in composite materials [1] since these nanocarbon materials possess extremely high Young's modulus and nearly theoretical mechanical strength. However, it is not an easy work to achieve toughening effects in ceramic composites, because it is technically difficult to disperse these nanomaterials uniformly in ceramic matrix. In addition, it is difficult to directly observe the interface, which is beyond the resolution of SEM while it is tedious to make sample for TEM observation, and not easy to get a true image of the interface [2]. Therefore, traditional trial-and-error method is not good enough in studying and developing nanocomposite materials. An alternative method is to use molecular dynamics to simulate the behavior of nanomaterials on atomic scale, thus give guidance for the design of nanocomposite before experiments [3]. As in conventional composite materials, the interface plays an essential role in the mechanical properties of nanocomposite. However, unlike the conventional composite, CNT or graphenes are atomically smooth, and the interface of CNT/ceramic would be van der Waals force, it is not effective to transfer stress from matrix to CNT.

In this research, we designed a branched CNT, and embedded it in SiC matrix, simulated the tensile behavior of the nanocomposite using molecular dynamics to see the effect of the branched CNT on the fracture process of nanocomposite.

\section{Materials and Simulation Method}

SiC block with a cubic crystal structure and a size of $5.12 \mathrm{~nm} \times 20.71 \mathrm{~nm} \times 216.9 \mathrm{~nm}$ was used in the simulation. A single-walled carbon nanotube (SWNT) $(6,6)$ with length of $16 \mathrm{~nm}$ was embedded in the $\mathrm{SiC}$ block to get a regular straight $\mathrm{CNT} / \mathrm{SiC}$ nanocomposite.

A branched CNT (Y-CNT) as shown in Fig. 1a was made in software VMD 1.9.3 [4] and Topotools [5]. The Y-CNT consisted of a straight SWNT $(6,6)$ with a length of $10 \mathrm{~nm}$ and Y-branches on two ends with a length of $1.5 \mathrm{~nm}$. The SiC block was drilled to allow the Y-CNT embedded in it. Since the interface between the SWNT and SiC was weak, the failure would occur in the interface between SiC and Y-branches under tensile load. To observe pullout of SWNT of Y-CNT from matrix, a large crack with a length of $2 \mathrm{~nm}$ and width of $1 \mathrm{~nm}$ was cut in the SiC block as shown in Fig. 1b. 
All simulations were performed by using the large-scale atomic molecular massively parallel simulator (LAMMPS) [6]. A hybrid potential, which includes $\mathrm{CH}$.airebo potential for C-C interaction in the CNT and Erhart/Albe-Tersoff potential for Si-C interaction in SiC and Lennard-Jones potential with $\sigma 0.038$ and $\varepsilon 2.96$ and cutoff $5.5 \AA$ for interaction between $\mathrm{SiC}$ and $\mathrm{CNT}$, was used to calculate the interatomic force. Initially, Brendsen thermostat was used to relax the system, so that the temperature fluctuates close to $300 \mathrm{~K}$.

During tensile loading, one end of the system was kept fixed, and the other end displaced in a strain rate of $0.02 \mathrm{~nm} / \mathrm{ps}$. The timestep was kept $1 \mathrm{fs}$ and the simulation was run up to 250,000 steps, which corresponding to 0.25 nanoseconds.

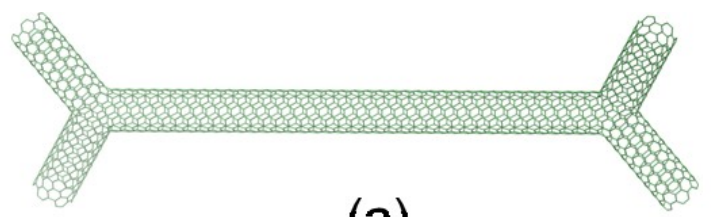

(a)

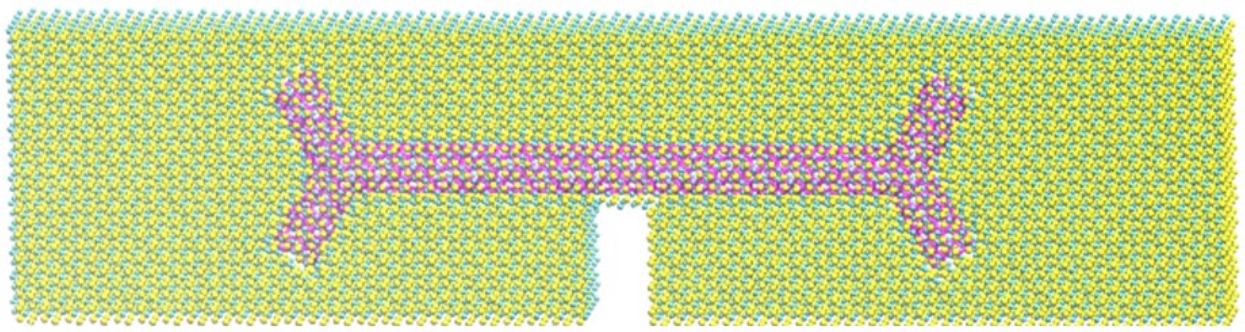

(b)

Fig. 1 (a) Y-branched CNT with length of $10 \mathrm{~nm}$ and branch length of $1.5 \mathrm{~nm}$. (b) Y-CNT was embedded in SiC block with a crack of $2 \mathrm{~nm}$ long and $1 \mathrm{~nm}$ wide.

\section{Results and Discussion}

The simulation of tensile test of $\mathrm{SiC}$ block without the $2 \mathrm{~nm}$ crack shows a smooth stress-strain curve. But if the crack was introduced in the SiC block, the stress-strain curve was not smooth, because an oscillation occurred on the sample, which was verified by the images and movie obtained from the simulation. The oscillation may be caused by the asymmetry of simulation box due to the crack. But the trend of stress-strain was similar to that of samples without introducing crack. If a straight SWNT was embedded in $\mathrm{SiC}$, the calculated stress-strain curve of $\mathrm{CNT} / \mathrm{SiC}$ was shown in Fig. 2. We can see that the straight-CNT/SiC composite shows lower Young's modulus and mechanical strength than

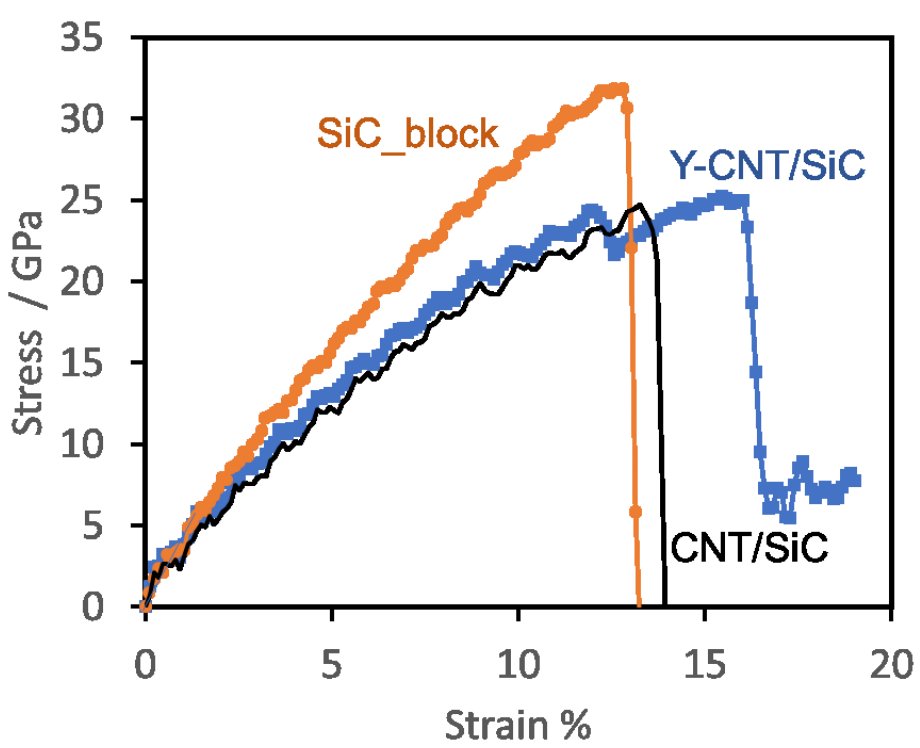

Fig. 2 The stress-strain curves of SiC-block, straight $\mathrm{CNT} / \mathrm{SiC}, \mathrm{Y}$-branched $\mathrm{CNT} / \mathrm{SiC}$ under tensile loading. All samples had a crack of $2 \mathrm{~nm}$ long.

SiC-block. Firstly, the interface between CNT and SiC was very weak, it is almost difficult for the stress transfer to CNTs. To form CNT/SiC nanocomposite, hollow space had to be formed in $\mathrm{SiC}$ 
block. In such small-scale nanocomposites, the original SiC had a nearly theoretical strength. Therefore, CNT into such matrix introduced defects into the matrix, resulting in decrease of Young's modulus and mechanical strength. However, the fracture strain of CNT/SiC was slightly larger than that of $\mathrm{SiC}$ block, suggesting a slight shielding effect of CNT to the propagation of the crack. In practical nanocomposite, the length of SWNT is in micrometers, it would be more effective in shielding the stress.

To allow CNT bearing stress effectively, some other mechanism is required. In this research, we proposed Y-branched CNT as shown in Fig. 1. Fig. 2 shows the stress-strain curve of Y-CNT/SiC. SiC-block shows a catastrophic failure at the strain of about $13 \%$. While the branched Y- CNT/SiC shows initially similar behavior to $\mathrm{SiC}$. But over $2 \%$, the slope of stress-strain curve of Y-CNT/SiC became smaller than that of $\mathrm{SiC}$, due to the deformation of the $\mathrm{Y}$-branches on the two ends, as shown in Fig. 3b. At the strain of $12.5 \%$ in the stress-strain curve of $\mathrm{Y}-\mathrm{CNT} / \mathrm{SiC}$, the stress dropped, due to a Y-branch deformation and pulling-into the tunnel of CNT. The deformed Y-branch was then pulled through the tunnel of CNT with further elongation of the Y-CNT/SiC. When the crack started to propagate through the $\mathrm{SiC}$, the Y-CNT bridged the crack, and CNT still bore stress through the sliding stress, and thus retarded the propagation of cracks, as illustrated in Fig. 3c. The sliding of Y-branch continued to the strain of about $16 \%$, where finally, the crack propagated through $\mathrm{SiC}$ and complete pullout occurred. After pullout, the Y-branch recovered to original shape as shown in Fig. 4d.

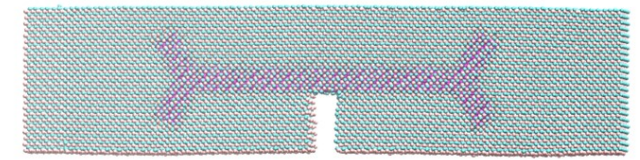

(a)

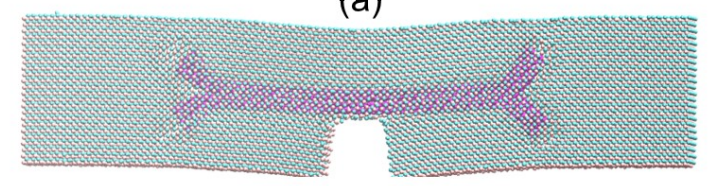

(b)

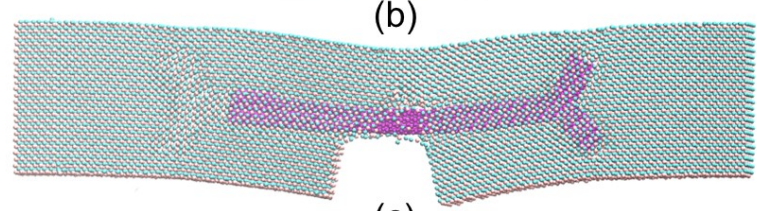

(c)

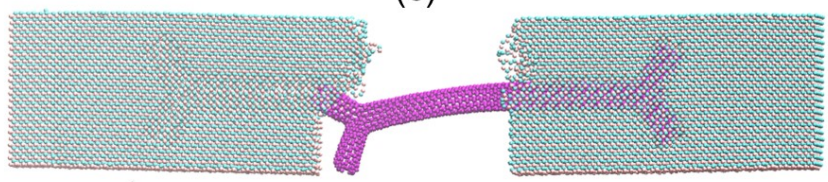

(d)

Fig. 3 The fracture process of $\mathrm{Y}-\mathrm{CNT} / \mathrm{SiC}$ nanocomposite under tensile load.

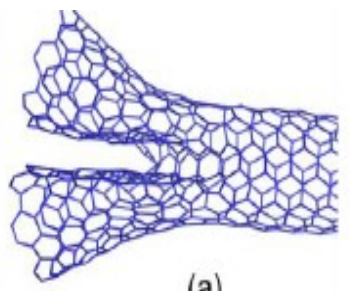

(a)

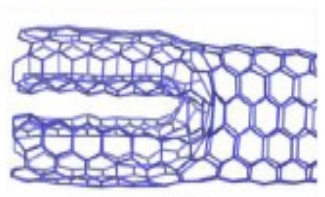

(b)

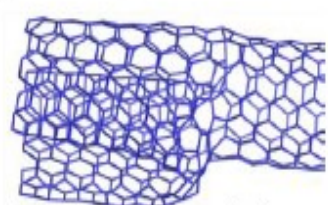

(c)

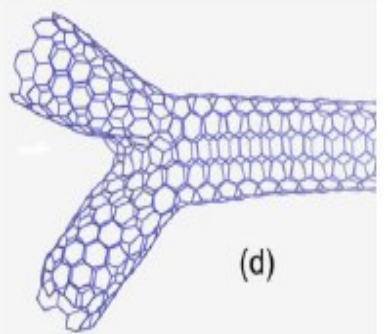

Fig. 4 The branch of CNT deformed during tensile loading of $\mathrm{Y}-\mathrm{CNT} / \mathrm{SiC}$ nanocomposite. (a) just before pulled into the tunnel. (b) (c) when pulled through the tunnel with different viewpoint, (d) recovered after complete pullout. 
Fig. 3 showed the snapshots of Y-branched CNT/SiC in different stages. Fig.3a was before stress loading but after relaxation. It was seen that the nanocomposite slightly bended downward, due to the existence of crack. With tensile loading, the Y-branches on the two ends of CNT inhibited the sliding of the CNT with matrix initially, but the Y-branches was under large stress, so it started to deform as shown in Fig. 3b. With increasing load, one branch was pulled into the tunnel of the CNT, even though the branch was larger than the hollow tunnel. After that, the deformed branch was pulled through the tunnel while further loading. Finally, complete pullout occurred, and the branch recovered to its original shape.

In Fig. 3, the deformation of CNT was not quite clearly identified. So, we showed the deformation of the CNT by hiding the atoms of SiC around the CNT, as shown in Fig. 4. Fig. 4a shows the CNT at the moment just before being pulled into the tunnel. Compared with the junction of the CNT in Fig. 1a, the carbon network in Fig. 4a deformed greatly, due to the flexibility of graphene structure. When the Y-branch was pulled into the tunnel of CNT, further deformation occurred on the Y-branch which changed to two parallel pipes (Fig. 4b), but in another viewpoint (Fig.4c) the compressed Y-branch was still larger than the diameter of straight CNT. Therefore, when the deformed Y-branch was pulled through the small space of CNT tunnel, the sliding stress would be quite large. After complete pullout, the deformed Y-branch back to the original shape without any damage.

According to the above result, we can see that the branched CNT was more effective to toughen the nanocomposite than a straight CNT. We also simulate SiC block with two Y-branched CNTs embedded, and similar results were obtained.

Kim et al simulated the tensile behavior of CNT/Si nanocomposite by changing the interface bonding between CNT and Si matrix [3]. It gave a direct effect of stress transfer on the mechanical properties of nanocomposites. However, strong bonding is difficult to form between atomically-smooth SWNT and Si without severely damaging SWNT. In practical case, CNTs are provided in the form of SWNT bundles or multiwalled CNTs, in which the interface between SWNTs in SWNT bundles or between walls in multiwalled CNTs is relatively weak, and thus sliding occurs easily so that effective stress transfer is not achieved [7]. Therefore, the branched CNT can effectively transfer the stress from matrix to CNT, at the same time, the crack does not propagate to CNT directly. The deformation of branch and pullout has a great contribution to the toughness of nanocomposite.

\section{Summary}

Simulations about the tensile behavior of CNT/SiC nanocomposite with a length of about $21.6 \mathrm{~nm}$ were performed. In such small simulation systems, the toughening effect of straight CNT was hardly achieved due to weak interface between CNT and the matrix. In Y-branched CNT/SiC nanocomposite, the branch of CNT inhibited the sliding of CNT and then the deformed branch of CNT was pulled into the tunnel of CNT, thus retarded the propagation of crack and improved the toughness of nanocomposites. MD can be a useful technique to gain an insight into the mechanical behavior occurred on atomic scale.

\section{References}

[1] Mittal G, Dhand V, Rhee KY, Park SJ, Lee WR, J. Ind. Eng. Chem. 21, 11-25 (2015).

[2] Yamamoto G, Omori M, Hashida T, Kimura H. Nanotechnology, 19(31), 315708, (2008). doi:10.1088/0957-4484/19/31/315708

[3] Kim BH, Lee KR, Chung YC, Lee G, J. Journal of Applied Physics, 112(4), 044312. (2012). doi:10.1063/1.4748133

[4] Humphrey W, Dalke A, Schulten K, J. Molec. Graphics, 14, 33-38. (1996)

[5] Kohlmeyer A, (2017). TopoTools, doi: 10.5281/zenodo.545655

[6] Plimpton S. J Comp Phys, 117, 1-19 (1995)

[7] Baughman RH, Zakhidov A A, Heer WA, Science, 297, 787-792 (2002). 